\title{
COMERCIALIZACIÓN DE MASA Y «FRUTO VERDE» DE AGUAJE (Mauritia flexuosa L.f.) EN IQUITOS (PERÚ)
}

Roberto Rojas Ruiz, Gabriel Ruiz Panduro, Pedro Ramírez Meléndez, Carlo F. Salazar Jarama, Cléver Rengifo Sias, Charles Llerena Flores, Camilo Marín Ríos, Dervin Torres Noriega, Julio Ojanama Vásquez, Wellington Silvano Alván, Vanessa Muñoz Isuiza, Hilter Luque Salinas, Nino Vela Gonza, Nelly del Castillo Fasabi, Jorge Solignac Ruiz, Víctor R. López de Oliveira, Flor de María Panduro Ruiz.*

\section{RESUMEN}

Este trabajo discute la comercialización de frutos de aguaje en forma de masa y «fruto verde» en la ciudad de Iquitos, Perú. Existen 11 mercados en la ciudad. En cuatro de ellos, es ofertada la masa; en dos, el «fruto verde» por 21 y 30 vendedoras, respectivamente. Ambas actividades son llevadas a cabo por mujeres mayores de 40 años, que tienen en promedio cuatro hijos y cuyas parejas, por lo general, no trabajan.

Las comercializadoras de masa y «fruto verde» se dedican a esta actividad desde hace 20 años en promedio. Cabe indicar, sin embargo, que la persona más experimentada la realiza desde hace ya 50 años. Las vendedoras ofrecen sus productos durante todo el año, lo que representa un consumo mensual de 3720 sacos, cantidad para la que se deben cortar aproximadamente 1078 palmeras.

De un saco de aguaje se obtienen en promedio 22 bolsas de masa y 16 bandejas pequeñas de «fruto verde». Esta cantidad rinde, en época de abundancia, utilidades superiores al salario mínimo de un obrero de la actividad privada. Asimismo, durante la época de escasez, el beneficio económico es mayor que el salario de un docente universitario estatal. Los frutos se exportan en cantidades mínimas a otras ciudades del Perú como Tarapoto, Pucallpa y Lima.

Las vendedoras han desarrollado técnicas sencillas y rápidas para conocer la madurez fisiológica del fruto y el ecotipo ofertado.

Palabras clave: Aguaje, Amazonía, burití, moriche, morete, canangucha.

* Círculo de Estudios e Investigación de Palmeras Amazónicas de la Facultad de Ingeniería Forestal de la Universidad Nacional de la Amazonía Peruana. 


\begin{abstract}
This work discusses the commercialization of fruits of «aguaje» in form of mass and «green fruit», in the city of Iquitos-Peru. Of the 11 markets of the city, the mass is sold in four and «green fruits» in two, existing 21 saleswomen of mass and 30 of «green fruit», both activities are dominated by women older than 40 years whose husbands mainly do not work and have in average four children.

They are in this activity during 20 years in average and the oldest person makes it for 50 years; $100 \%$ sell these products all the year, that represents a monthly consumption of 3720 sacks, equivalent to cut 1078 palm trees.

A sack of aguaje contains in average 22 bags of mass and 16 trays of «green fruit», that it renders utilities superior to the minimum of the wage of a worker of the private activity and at time of shortage superior to an educational state college teacher; one exports minimum amounts to other cities of Peru like Tarapoto, Pucallpa and Lima. They have developed simple and fast technics to know the physiological maturity of the fruit and ecotipe supplied.
\end{abstract}

Key words: Aguaje, Amazon, burití, moriche, morete, canangucha.

\title{
1. INTRODUCCIÓN
}

El aguaje (Mauritia flexuosa L.f.) es, sin duda, el producto forestal diferente de la madera más importante en la vida económica de Iquitos. A pesar de tener una amplia distribución en todo el norte de Sudamérica y al este de los Andes (Henderson, 1995; Henderson, Galeano y Bernal, 1995), el comercio a gran escala solo se observa en Iquitos (Perú) y, en pequeña escala, en el Ecuador (Ojeda, 1994).

El primer estudio amplio sobre la importancia económica del aguaje en Iquitos lo realizó Padoch (1988). Desde ese año hasta ahora, no existe otro estudio que haya profundizado en el tema para conocer en mayor detalle su importancia.

Las publicaciones de Ruiz (1991, 1993) destacan la importancia del aguaje como alimento y plantean la tesis de que no solo la madera sino también los alimentos del bosque son los nuevos conceptos que deben guiar la actividad forestal en los próximos decenios. De esta manera, se frenaría la deforestación y se lograría la preservación y conservación de los bosques. 
La importancia del aguaje como alimento y proveedor de otros productos no es un descubrimiento reciente, puesto que ya en 1852 el célebre científico alemán Alexander von Humboldt lo llamó «árbol de vida» (Storti, 1993) y el coautor de la teoría de la evolución, Alfred Russel Wallace, impresionado por la extensión de las poblaciones naturales de aguaje, escribió en 1853 acerca de «un vasto templo natural que no palidece en grandeza y sublimidad frente a aquel compuesto por la Palmyra de Atenas» (Balick, 1979).

Es preocupante la falta de estudios sobre esta especie. Y resulta más preocupante aún si es que los cálculos realizados demuestran que, mensualmente, en Loreto se cortan 1000 plantas femeninas de aguaje en los aguajales existentes en las diferentes cuencas de los ríos donde abunda la especie (Rojas et al., 2001).

En este trabajo, estudiamos la comercialización de los frutos del aguaje como «aguaje verde» y como masa, dos aspectos importantes de la cadena de comercialización de los frutos de esta palmera. Con ello, esperamos contribuir al mejor conocimiento del importante papel de esta especie en la vida económica de Iquitos y, a la vez, saber los pormenores de estas actividades para que sirvan de inicio a investigaciones posteriores.

\section{MATERIALES Y MÉTODOS}

Este estudio se realizó en la ciudad de Iquitos (3 45' 05" S y 73 14' 40” O; 122,4 msnm), capital del departamento de Loreto, en el nordeste del Perú. Climáticamente, dicha ciudad se ubica dentro del tipo de clima A(r) A' H4, caracterizado por ser muy lluvioso; la zona de vida que predomina es la del Bosque Húmedo Tropical (Flores, Gómez y Kalliola, 1998).

Según Marengo (1998), la temperatura media es de $26^{\circ} \mathrm{C}$; la máxima, de $30{ }^{\circ} \mathrm{C}$ y la mínima, de $22{ }^{\circ} \mathrm{C}$; la precipitación mensual, de $257 \mathrm{~mm}$; la anual, de $3087 \mathrm{~mm}$; la humedad relativa máxima, de $95 \%$ y humedad mínima, de $74 \%$.

Se distinguen claramente dos estaciones: una seca llamada verano, que se extiende desde junio hasta octubre, que es el periodo de estiaje de los ríos, y otra lluviosa llamada invierno, desde noviembre hasta mayo, que es el periodo de inundaciones o crecida de los ríos.

El levantamiento de la información se realizó por medio de encuestas estructuradas con entrevistas directas a todos los vendedores de masa y «fruto verde» de aguaje en 
todos los mercados y mercadillos de la ciudad. Luego de procesada la información, se elaboraron tablas para el análisis de las mismas.

En Iquitos se conoce como masa de aguaje al producto obtenido luego de ablandar, machacar y eliminar las semillas y gran parte de la cáscara del mesocarpo del fruto maduro. De otro lado, el «fruto verde» o «aguaje verde» es el fruto fisiológicamente maduro que no ha sido sometido al proceso de ablandamiento del mesocarpo. Cuando la cáscara es todavía verde, está fuertemente adherida al mesocarpo y este último es relativamente duro.

\section{RESULTADOS Y DISCUSIÓN}

\subsection{Mercados y mercadillos de la ciudad de Iquitos}

En Iquitos, existen 11 mercados para atender a una población superior a 231648 habitantes (INEI, 1993). Las categorías de mercado y mercadillo se deben, fundamentalmente, al tipo de infraestructura y al número de vendedores y servicios básicos con que se cuenta. Mientras que los mercados tienen áreas techadas, construidas con ladrillo y cemento, y servicios de agua y luz; los mercadillos, generalmente, no los tienen. Únicamente dos mercadillos poseen estos servicios: La Norteñita y San Juan.

El mercado Belén, ubicado al sur de la ciudad, es el más importante, ya que es el más grande, cuenta con el mayor número de vendedores y oferta toda clase de productos a bajos precios. Jerárquicamente, el mercado Modelo, ubicado al norte de la ciudad, está colocado luego del Belén. Por su parte, La Norteñita, ubicado al oeste, es el más representativo de los mercadillos (Tabla 1).

Puerto Masusa no es propiamente un mercado ni un mercadillo, pues es el lugar de llegada y salida de embarcaciones fluviales de diferentes ríos de Loreto. Sin embargo, dadas las condiciones, allí se ubican vendedores y compradores de diferentes productos, y, desde la perspectiva de nuestro estudio, allí existen numerosos comerciantes mayoristas de frutos de aguaje. Por ello, lo consideramos como un mercado más. 
Tabla 1: Mercados y mercadillos de la ciudad de Iquitos.

\begin{tabular}{lllr}
\hline N $^{\circ}$ & \multicolumn{1}{c}{ Nombre } & \multicolumn{1}{c}{ Ubicación } & $\begin{array}{r}\mathbf{N}^{\circ} \text { total de } \\
\text { vendedores }\end{array}$ \\
\hline 1 & Mercado Belén & $\begin{array}{l}\text { Calle Ramírez Hurtado, García } \\
\text { Sáenz, J.C. Arana, 9 de }\end{array}$ & 2364 \\
& & $\begin{array}{l}\text { Diciembre, Abato, Alfonso } \\
\text { Ugarte, Gálvez }\end{array}$ & \\
& & Calle Sargento Lores - Moore & 1286 \\
2 & Mercado Modelo & Calle Celendín - Nanay - Arequipa & 256 \\
3 & Mercado Central & Calle Trujillo - Iquitos - 3 de Octubre & 192 \\
4 & Mercado Clavero & Avenida del Ejército & 70 \\
5 & Mercado Morona Cocha & 26 \\
6 & Mercado de Productores & Calle Requena & 12 \\
7 & Mercadillo San Juan & Avenida Quiñones km 4 & 97 \\
8 & Mercadillo Túpac Amaru & Calle Lourdes de León - Jesús Paenz & 245 \\
9 & Mercadillo La Norteñita & Calle Putumayo - Manco Cápac & 60 \\
10 & Mercadillo Bellavista Nanay & Orillas río Nanay & 136 \\
11 & Puerto Masusa & Orillas río Amazonas & \\
\hline
\end{tabular}

\subsection{Vendedoras de masa y «fruto verde» de aguaje en Iquitos}

En Iquitos existen 21 personas que ofertan masa de aguaje y 30 que ofertan «fruto verde». La mayor cantidad de vendedoras de «fruto verde» indicaría una mayor preferencia del público por los frutos del aguaje, que el comprador mismo puede hacer madurar para luego consumir. De esta manera, el público tendría la oportunidad de escoger los frutos de su preferencia.

En cuatro de los 11 centros de abasto de la ciudad se oferta masa de aguaje; en dos, «fruto verde». El mercado Belén ostenta el mayor porcentaje de vendedoras para ambos productos (67\% para masa y $77 \%$ para «fruto verde»). A este le sigue el mercado Modelo con $14 \%$ para masa y $23 \%$ para «fruto verde» (Tabla 2 ).

En el caso del mercado Belén, las vendedoras de masa representan el 0,59\% del total de vendedores del mercado, mientras que las de «fruto verde» representan el $0,97 \%$. Por lo que respecta al mercado Modelo, las comerciantes de masa representan el $0,23 \%$ y las de «fruto verde», el $0,54 \%$ de la cantidad total de vendedores del mercado. 
En general, se puede afirmar que las vendedoras de masa y «fruto verde» de aguaje no alcanzan el $1 \%$ del total de vendedores en los mercados donde se ofertan estos productos.

Tabla 2: Vendedoras de masa y «fruto verde» de aguaje/mercados.

\begin{tabular}{lcccc}
\hline \multicolumn{1}{c}{ Mercado } & $\begin{array}{c}\mathbf{N}^{\circ} \text { de } \\
\text { vendedoras de } \\
\text { masa }\end{array}$ & \% & $\begin{array}{c}\mathbf{N}^{\circ} \text { de } \\
\text { vendedoras } \\
\text { de «fruto verde» } \\
\text { de aguaje }\end{array}$ & $\%$ \\
\hline Mercado Belén & 14 & 66,67 & 23 & 76,67 \\
Mercado Modelo & 3 & 14,29 & 7 & 23,33 \\
Mercadillo La Norteñita & 2 & 9,52 & 0 & - \\
Mercado Clavero & 1 & 4,76 & 0 & - \\
Mercado Morona Cocha & 1 & 4,76 & 0 & - \\
Mercado Central & 0 & - & 0 & - \\
Mercado de Productores & 0 & - & 0 & - \\
Mercadillo San Juan & 0 & - & 0 & - \\
Mercadillo Túpac Amaru & 0 & - & 0 & - \\
Mercadillo Bellavista Nanay & 0 & - & 0 & - \\
Puerto Masusa & 0 & - & 0 & - \\
\hline TOTAL & 21 & 100,00 & 30 & 100,00 \\
\hline
\end{tabular}

\subsection{Datos de las vendedoras de masa y «fruto verde» de aguaje}

La totalidad de las personas que venden masa de aguaje son mujeres, mientras que el 93\% de los vendedores de «fruto verde» está representado por mujeres y el 7\%, por hombres (Tablas 3 y 4). Así se demuestra que estas actividades son eminentemente femeninas, lo que concuerda con las observaciones realizadas por Padoch (1988).

Una explicación a este hecho puede encontrarse en los patrones machistas de la sociedad ribereña y de la clase pobre de Iquitos: por tratarse de actividades que no requieren de un gran esfuerzo físico, los hombres las consideran actividades propias del sexo femenino. 
Tabla 3: Datos de vendedoras de masa en la ciudad de Iquitos.

\begin{tabular}{|c|c|c|c|c|c|c|}
\hline Mercado & Sexo & Edad & $\begin{array}{c}\text { Estado } \\
\text { civil }\end{array}$ & $\begin{array}{l}\text { Número } \\
\text { de hijos }\end{array}$ & $\begin{array}{l}\text { Hijos que } \\
\text { estudian }\end{array}$ & $\begin{array}{l}\text { Ocupación de } \\
\text { la pareja }\end{array}$ \\
\hline \multirow[t]{14}{*}{ Belén } & $\mathrm{F}$ & 35 & Conviviente & 5 & 4 & Barredor \\
\hline & $\mathrm{F}$ & 52 & Viuda & 6 & 1 & - \\
\hline & $\mathrm{F}$ & 25 & Soltera & 1 & 0 & - \\
\hline & $\mathrm{F}$ & 30 & Conviviente & 4 & 3 & Rematista \\
\hline & $\mathrm{F}$ & 28 & Conviviente & 3 & 2 & Vendedor \\
\hline & $\mathrm{F}$ & 42 & Casada & 4 & 2 & Ambulante \\
\hline & $\mathrm{F}$ & 31 & Casada & 2 & 2 & Albañil \\
\hline & $\mathrm{F}$ & 67 & Viuda & 6 & 1 & - \\
\hline & $\mathrm{F}$ & 66 & Casada & 7 & 0 & No trabaja \\
\hline & $\mathrm{F}$ & 66 & Casada & 12 & 5 & Agricultor \\
\hline & $\mathrm{F}$ & 25 & Conviviente & 1 & 0 & Obrero \\
\hline & $\mathrm{F}$ & 47 & Casada & 2 & 2 & Motocarrista \\
\hline & $\mathrm{F}$ & 34 & Conviviente & 5 & 5 & Eventual \\
\hline & $\mathrm{F}$ & 27 & Casada & 2 & 1 & $\begin{array}{c}\text { Trabaja con } \\
\text { la esposa }\end{array}$ \\
\hline \multirow[t]{3}{*}{ Modelo } & $\mathrm{F}$ & 30 & Conviviente & 3 & 2 & Peluquero \\
\hline & $\mathrm{F}$ & 59 & Soltera & 5 & 5 & No trabaja \\
\hline & $\mathrm{F}$ & 47 & Casada & 5 & 2 & Timonel \\
\hline \multirow[t]{2}{*}{ La Norteñita } & $\mathrm{F}$ & 50 & Casada & 8 & 3 & Obrero \\
\hline & $\mathrm{F}$ & 28 & Soltera & 0 & 0 & - \\
\hline Clavero & $\mathrm{F}$ & 60 & Casada & 7 & 0 & Albañil \\
\hline Morona Cocha & $\mathrm{F}$ & 53 & Casada & 4 & 4 & Eventual \\
\hline
\end{tabular}

La edad promedio de las vendedoras de masa es de 43 años. Sus edades varían dentro de un rango que oscila entre los 25 y 67 años. Por su parte, las vendedoras de «fruto verde» tienen, en promedio, 48 años de edad, y sus edades varían desde los 29 hasta los 82 años. Se observa claramente que las vendedoras de masa son, en promedio, más jóvenes que las vendedoras de «fruto verde», lo cual puede explicarse por el mayor esfuerzo físico involucrado en la preparación de la masa.

La estratificación de edades de las vendedoras de masa se divide de la siguiente manera: el $43 \%$ se ubica entre los 25 y 35 años; el 10\%, entre los 36 y 45 años; el $23 \%$, entre los 46 y 55 años; el $10 \%$, entre los 56 y 65 años; y el 14\%, entre los 76 y 85 años. A su vez, el $23 \%$ de las vendedoras de «fruto verde» se ubica entre 25 y 35 ; otro $23 \%$, entre 36 y 45 años; el $27 \%$, entre los 46 y 55 años; el $10 \%$, entre los 56 y 65 años; el 13\%, entre los 66 y 75 años; y el 3\%, entre los 76 y 85 años. 
Tabla 4: Datos de vendedoras de «fruto verde» en la ciudad de Iquitos.

\begin{tabular}{|c|c|c|c|c|c|c|}
\hline Mercado & Sexo & Edad & $\begin{array}{c}\text { Estado } \\
\text { civil }\end{array}$ & $\begin{array}{l}\text { Número } \\
\text { de hijos }\end{array}$ & $\begin{array}{r}\text { Hijos que } \\
\text { estudian }\end{array}$ & $\begin{array}{l}\text { Ocupación de } \\
\text { la pareja }\end{array}$ \\
\hline Belén & $\mathrm{F}$ & 35 & Conviviente & 5 & 4 & Barredor \\
\hline \multirow[t]{23}{*}{ Belén } & $\mathrm{F}$ & 55 & Soltera & 4 & - & - \\
\hline & $\mathrm{F}$ & 39 & Casada & 6 & 3 & Obrero \\
\hline & $\mathrm{F}$ & 32 & Conviviente & 5 & 5 & $\begin{array}{c}\text { Trabaja con la } \\
\text { esposa }\end{array}$ \\
\hline & $\mathrm{F}$ & 51 & Divorciada & 8 & 1 & - \\
\hline & $\mathrm{F}$ & 36 & Conviviente & 4 & 2 & Vendedor de frutas \\
\hline & $\mathrm{F}$ & 66 & Casada & 7 & - & No trabaja \\
\hline & $\mathrm{F}$ & 82 & Viuda & 9 & - & - \\
\hline & $\mathrm{F}$ & 29 & Conviviente & 2 & 2 & $\begin{array}{l}\text { Extractor de } \\
\text { madera }\end{array}$ \\
\hline & $\mathrm{F}$ & 34 & Conviviente & 1 & 1 & Motorista \\
\hline & $\mathrm{F}$ & 48 & Conviviente & 8 & - & No trabaja \\
\hline & $\mathrm{F}$ & 65 & Conviviente & 8 & - & $\begin{array}{c}\text { Trabaja con la } \\
\text { esposa }\end{array}$ \\
\hline & $\mathrm{F}$ & 58 & Soltera & 0 & - & - \\
\hline & $\mathrm{F}$ & 33 & Casada & 1 & 1 & $\begin{array}{c}\text { Trabaja con la } \\
\text { esposa }\end{array}$ \\
\hline & $\mathrm{F}$ & 41 & Casada & 4 & 2 & Ambulante \\
\hline & $\mathrm{F}$ & 67 & Viuda & & 1 & - \\
\hline & $\mathrm{F}$ & 49 & Soltera & 8 & 5 & - \\
\hline & $\mathrm{F}$ & 60 & Soltera & 7 & 1 & - \\
\hline & $\mathrm{M}$ & 39 & Soltero & 2 & 2 & - \\
\hline & $\mathrm{F}$ & 74 & Viuda & 4 & - & - \\
\hline & $\mathrm{F}$ & 35 & Conviviente & 5 & 3 & Albañil \\
\hline & $\mathrm{F}$ & 30 & Conviviente & 1 & 1 & No trabaja \\
\hline & $\mathrm{F}$ & 30 & Divorciada & 5 & - & Ambulante \\
\hline & $\mathrm{F}$ & 44 & Conviviente & 3 & 3 & No trabaja \\
\hline \multirow[t]{7}{*}{ Modelo } & $\mathrm{F}$ & 41 & Conviviente & 4 & 3 & $\begin{array}{c}\text { Trabaja con la } \\
\text { esposa }\end{array}$ \\
\hline & $\mathrm{F}$ & 68 & Soltera & 7 & - & - \\
\hline & $\mathrm{F}$ & 40 & Conviviente & 4 & 1 & $\begin{array}{c}\text { Trabaja con la } \\
\text { esposa }\end{array}$ \\
\hline & $\mathrm{F}$ & 48 & Soltera & 6 & - & - \\
\hline & $\mathrm{F}$ & 48 & Conviviente & 6 & 2 & $\begin{array}{c}\text { Trabaja con la } \\
\text { esposa }\end{array}$ \\
\hline & $\mathrm{F}$ & 50 & Viuda & 6 & - & - \\
\hline & $\mathrm{M}$ & 46 & Conviviente & 1 & - & No trabaja \\
\hline
\end{tabular}


En cuanto al estado civil, se observa que el $48 \%$ de las vendedoras de masa se encuentra casado y el $29 \%$ convive, mientras que, entre las vendedoras de «fruto verde», solo el $13 \%$ está casado y el $43 \%$ es conviviente. Lo anterior indica que, entre las vendedoras de masa de aguaje, existe una mayor estabilidad jurídica matrimonial, que se corrobora con las cifras de vendedoras solteras en ambos rubros: $14 \%$ en las vendedoras de masa y $23 \%$ en las vendedoras de «fruto verde».

El mayor número de viudas (13\%) y de divorciadas (7\%) entre las vendedoras de «fruto verde» se explica por su mayor rango de edad (29 - 82) en comparación con el de las vendedoras de masa (25 - 67).

El número promedio de hijos entre las vendedoras de masa es cuatro. En este caso, el rango oscila desde 0 hasta 12 hijos. El 38\% de las madres tiene entre 0 y 3 hijos; y el $48 \%$, entre 4 y 7 . Por lo que respecta a las vendedoras de «fruto verde», el número promedio de hijos es cinco y el rango varía desde ninguno hasta 9 hijos. El 27\% de madres tiene entre 0 y 3 hijos; el 57\%, entre 4 y 7 hijos. El mayor número de hijos entre las vendedoras de «fruto verde» también se explica por el mayor rango de edad que existe en este grupo.

En ambos grupos, todos los hijos que están en edad escolar estudian. Cabe resaltar el caso de una madre de cuatro hijos, de los cuales tres asisten a la universidad y uno al Instituto Tecnológico, a pesar de que su padre solo tiene un trabajo eventual. Se confirma así que esta actividad de venta de aguaje provee los suficientes ingresos económicos para satisfacer las necesidades básicas de educación y otras, tal como lo mencionara Padoch (1988).

De las 30 vendedoras de «fruto verde», 19 tienen pareja (ya sea que estén casadas o convivan). Seis de estas parejas no tienen trabajo e igual número de ellas trabaja junto con su esposa. Estas cifras representan, en conjunto, el 63\% de los desempleados. Por lo que respecta a las siete parejas restantes (37\%), estas tienen ocupaciones eventuales que no aseguran un aporte fijo al ingreso familiar. De otro lado, por lo que concierne al grupo de vendedoras de masa: de las 21 vendedoras, 18 tienen parejas (con las que están casadas o conviven). Dos de estas parejas no trabajan, mientras que una trabaja con su mujer. En conjunto, las cifras mencionadas representan el $17 \%$. En lo que toca a las 15 restantes parejas (83\%), estas tienen ocupaciones similares a las del primer grupo. Por ello, se puede afirmar que el peso del mantenimiento familiar recae en las mujeres vendedoras de masa y «fruto verde». Estos datos también confirman las afirmaciones de Padoch (1988). 


\subsection{Antigüedad en el negocio, lugares y precio de compra de los sacos de aguaje}

Es sorprendente comprobar que en este negocio exista una vinculación de muchos años. Las vendedoras de masa están en el negocio 21 años en promedio con un rango de variabilidad entre 4 a 48 años. El 66\% de ellas tiene entre 1 y 25 años dedicándose a esta actividad; el $34 \%$, entre 26 y 50 años. A su vez, las vendedoras de «fruto verde» están en el negocio 19 años en promedio. En este caso, el rango de variabilidad oscila entre 1 y 50 años. El $73 \%$ de ellas tiene entre 1 y 25 años en esta actividad; el $27 \%$, entre 26 a 50 años. En la práctica, se puede afirmar que el promedio de años dedicados a este trabajo es igual para ambos casos (Tablas 5 y 6 ).

Tabla 5: Antigüedad en el negocio, lugares y precio de compra de la materia prima y ventas diarias de masa.

\begin{tabular}{|c|c|c|c|c|c|c|c|c|}
\hline \multirow[t]{2}{*}{ Mercado } & \multirow[t]{2}{*}{$\mathbf{A N}$} & \multirow[t]{2}{*}{$\mathrm{D} / \mathrm{S}$} & \multirow[t]{2}{*}{$\begin{array}{l}\text { Lugar de compra } \\
\text { de sacos }\end{array}$} & \multicolumn{2}{|c|}{$\begin{array}{c}\text { Precio } \\
\text { de } \operatorname{saco}(\mathrm{S} / .)\end{array}$} & \multirow[t]{2}{*}{ SVD } & \multirow[t]{2}{*}{$\mathbf{S} / \mathbf{M}$} & \multirow[t]{2}{*}{$\begin{array}{c}\text { Otros } \\
\text { productos }\end{array}$} \\
\hline & & & & $\mathbf{A}$ & $\mathbf{E}$ & & & \\
\hline \multirow[t]{14}{*}{ Belén } & 12 & 7 & Belén & 10 & 20 & 2 & 2 & - \\
\hline & 20 & 7 & Belén & 10 & 60 & 4 & 4 & - \\
\hline & 10 & 7 & Belén & 7 & 20 & 2 & 2 & - \\
\hline & 4 & 7 & Masusa-Lanchas & 8 & 35 & 4 & 4 & - \\
\hline & 6 & 7 & Belén & 18 & 35 & 4 & 2 & $\mathrm{fm}$ \\
\hline & 28 & 7 & Belén-Masusa-Lanchas & 8 & 50 & 2 & 2 & - \\
\hline & 4 & 7 & Belén-Lanchas & 10 & 30 & 2 & 1 & $\mathrm{fm}-\mathrm{a}$ \\
\hline & 47 & 7 & Belén & 7 & 18 & 1 & 0,5 & $\mathrm{fm}$ \\
\hline & 48 & 7 & Belén & 8 & 35 & 1 & 0,5 & $\mathrm{fm}-\mathrm{a}$ \\
\hline & 16 & 7 & Belén & 5 & 50 & 2 & 1 & $\mathrm{fm}-\mathrm{a}$ \\
\hline & 20 & 7 & Masusa & 4 & 50 & 2 & 1 & fm-a-c \\
\hline & 10 & 7 & Masusa & 5 & 40 & 6 & 5 & $a-c$ \\
\hline & 20 & 7 & Masusa & 8 & 50 & 7 & 5,5 & fm \\
\hline & 8 & 7 & Masusa & 7 & 30 & 2 & 2 & - \\
\hline \multirow[t]{3}{*}{ Modelo } & 30 & 7 & Masusa & 8 & 30 & 4 & 3 & $\mathrm{c}$ \\
\hline & 30 & 7 & Masusa-Lanchas-Product. & 5 & 20 & 2 & 1 & fm-a \\
\hline & 15 & 7 & Masusa-Lanchas & 10 & 50 & 5 & 4 & fv-fm-a \\
\hline \multirow[t]{2}{*}{ La Norteñita } & 7 & 4 & Masusa-Belén & 9 & 20 & 4 & 1 & fm-a \\
\hline & 36 & 7 & Masusa & 13 & 35 & 3 & 2 & $\mathrm{fm}-\mathrm{c}$ \\
\hline Clavero & 20 & 7 & Masusa-Belén-Product. & 5 & 35 & 2 & 1 & $\mathrm{fm}-\mathrm{a}$ \\
\hline Morona Cocha & 40 & 7 & Masusa-Lanchas & 18 & 30 & 2 & 1 & $\mathrm{fm}-\mathrm{a}$ \\
\hline
\end{tabular}

$\mathrm{AN}=$ Años en el negocio, $\mathrm{D} / \mathrm{S}=$ Días de venta en la semana, $\mathrm{A}=$ Abundancia, $\mathrm{E}=$ Escasez, $\mathrm{SVD}=\mathrm{Sacos}$ vendidos por día, $\mathrm{S} / \mathrm{M}=$ Sacos convertidos en masa, $\mathrm{fm}=$ Fruto maduro, $\mathrm{fv}=$ Fruto verde, $\mathrm{a}=$ Aguajina, $\mathrm{c}=$ Curichi. 
Tanto la venta de masa como la de «fruto verde» son realizadas los siete días de la semana y de manera exclusiva para productos de aguaje. Más claramente, en el caso de «fruto verde», esta se realiza exclusivamente y, en el caso de masa, cuando esta sobra, se vende transformada como «fruta madura», aguajina o curichi, pues cuando la pulpa es expuesta al medio ambiente se oxida rápidamente y pierde sus cualidades (Rojas et al., 2001). Además, en tanto que estos últimos son productos diferentes a la masa, las vendedoras obtienen mayores ganancias, aunque para ello necesiten estar más tiempo en el lugar de venta.

En cuanto al lugar de compra de la materia prima (sacos de aguaje), según la Tabla 5 se observa que, de las 14 vendedoras de masa de aguaje del mercado Belén, 7 la compran exclusivamente a los mayoristas de aguaje del mismo mercado, una lo hace, ya sea a los mayoristas de Belén, ya sea a las lanchas, mientras que otra compra en los mayoristas de Belén, en las lanchas o en Puerto Masusa. Las 5 restantes compran solo en Puerto Masusa. Por lo que respecta a las 7 vendedoras de los demás mercados, estas adquieren su materia prima en Puerto Masusa.

El hecho de que el 69\% de vendedoras de masa del mercado Belén compre su materia prima a los mayoristas del mismo mercado se explica por la menor distancia y la facilidad del transporte a sus puestos de venta. Que el 31\% restante compre en Puerto Masusa puede deberse a los precios más bajos que se ofertan en ese lugar y a su mayor capacidad adquisitiva. Tal como puede observarse en la misma Tabla, son 5 las vendedoras que compran el mayor número de sacos.

Las vendedoras de los demás mercados, generalmente, compran la materia prima en Puerto Masusa por la cercanía al mercado donde trabajan. Sin embargo, muchas de ellas también hacen sus compras en Belén y en el Mercado de Productores.

En cuanto al lugar de compra de las vendedoras de «fruto verde», según la Tabla 6, se observa que, de las 23 vendedoras del mercado Belén, $48 \%$ compra la materia prima exclusivamente a los mayoristas del mismo mercado; $13 \%$ lo hace exclusivamente en Puerto Masusa; $6 \%$ hace sus compras, ya sea a los mayoristas de Belén, ya sea directamente a las lanchas; mientras que $3 \%$ obtiene sus materias primas gracias a los mayoristas o en Puerto Masusa. Por lo que respecta a las 7 vendedoras del mercado Modelo, 57\% realiza sus compras exclusivamente en Puerto Masusa y 43\% en Puerto Masusa o en el Mercado de Productores.

La preferencia por el lugar de compra se debe a la cercanía del centro de abasto, pues, en el caso de las vendedoras del Mercado Modelo, su centro de trabajo y el de abastecimiento se encuentran a poca distancia. Ocurre lo mismo con las vendedoras del mercado Belén. El hecho de que se observe que algunas vendedoras realizan también 
sus compras en Puerto Masusa o directamente en las lanchas se debe, probablemente, al mayor poder adquisitivo de estas y a la búsqueda de menores precios por los sacos.

Tabla 6: Antigüedad en el negocio, lugares de compra, precio de compra de la materia prima y ventas diarias de «fruto verde».

\begin{tabular}{|c|c|c|c|c|c|c|c|}
\hline \multirow[t]{2}{*}{ Mercado } & \multirow{2}{*}{$\begin{array}{c}\text { Años } \\
\text { en el } \\
\text { negocio }\end{array}$} & \multirow{2}{*}{$\begin{array}{c}\text { Días } \\
\text { semana } \\
(1)\end{array}$} & \multirow[t]{2}{*}{$\begin{array}{l}\text { Lugar de compra } \\
\text { de sacos }\end{array}$} & \multicolumn{2}{|c|}{$\begin{array}{c}\text { Precio } \\
\text { de saco }\end{array}$} & \multirow{2}{*}{$\begin{array}{c}\text { Sacos } \\
\text { vendidos } \\
\text { x día }\end{array}$} & \multirow{2}{*}{$\begin{array}{c}\text { Sacos } \\
\text { s vendidos } \\
\text { como fruto } \\
\text { verde }\end{array}$} \\
\hline & & & & $\begin{array}{l}\mathbf{A} \\
\text { (2) }\end{array}$ & $\begin{array}{l}\mathbf{E} \\
\text { (3) }\end{array}$ & & \\
\hline \multirow[t]{23}{*}{ Belén } & 15 & 7 & Belén- Masusa & 15 & 50 & 1 & 1 \\
\hline & 7 & 7 & Belén-Lanchas & 15 & 40 & 3 & 3 \\
\hline & 4 & 7 & Belén-Lanchas & 5 & 30 & 8 & 8 \\
\hline & 15 & 7 & Belén-Lanchas & 10 & 50 & 3 & 3 \\
\hline & 7 & 7 & Belén-Lanchas & 12 & 50 & 7 & 7 \\
\hline & 50 & 7 & Belén-Lanchas & 8 & 50 & 1 & 1 \\
\hline & 25 & 7 & Belén-Lanchas & 15 & 30 & 2 & 2 \\
\hline & 10 & 7 & Belén-Masusa & 10 & 60 & 2 & 2 \\
\hline & 10 & 7 & Belén & 20 & 60 & 3 & 3 \\
\hline & 26 & 7 & Masusa & 15 & 30 & 1 & 1 \\
\hline & 35 & 7 & Belén & 15 & 30 & 1 & 1 \\
\hline & 25 & 7 & Belén & 7 & 20 & 1 & 1 \\
\hline & 20 & 7 & Masusa & 15 & 35 & 2 & 2 \\
\hline & 27 & 7 & Belén-Masusa & 8 & 50 & 2 & 2 \\
\hline & 47 & 7 & Belén & 5 & 30 & 1 & 1 \\
\hline & 29 & 7 & Belén & 7 & 50 & 1 & 1 \\
\hline & 3 & 7 & Belén & 15 & 30 & 1 & 1 \\
\hline & 20 & 7 & Belén & 8 & 70 & 2 & 2 \\
\hline & 35 & 7 & Belén & 10 & 20 & 2 & 2 \\
\hline & 12 & 7 & Belén & 12 & 20 & 2 & 2 \\
\hline & 3 & 7 & Belén & 15 & 25 & 1 & 1 \\
\hline & 20 & 7 & Belén & 6 & 20 & 1 & 1 \\
\hline & 8 & 7 & Masusa & 7 & 20 & 3 & 3 \\
\hline \multirow[t]{7}{*}{ Modelo } & 10 & 7 & Masusa & 8 & 60 & 1 & 1 \\
\hline & 1 & 7 & Masusa & 8 & 60 & 2 & 2 \\
\hline & 2 & 7 & Masusa & 8 & 25 & 1 & 1 \\
\hline & 6 & 7 & Masusa & 15 & 60 & 1 & 1 \\
\hline & 25 & 7 & Masusa-Productores & 8 & 40 & 1 & 1 \\
\hline & 18 & 7 & Masusa-Productores & 5 & 40 & 1 & 1 \\
\hline & 40 & 7 & Masusa-Productores & 5 & 50 & 3 & 3 \\
\hline
\end{tabular}

(1) : Días de venta en la semana, (2) : Abundancia, (3) : Escasez. 
En época de abundancia, las vendedoras de masa pagan, en promedio, 9 soles por saco de aguaje. El rango de este costo varía desde 4 hasta 18 soles. En época de escasez, se paga 37 soles en promedio y la variación va desde 18 hasta 60 soles. En cuanto a las vendedoras de «fruto verde», ellas pagan 10 soles por saco en promedio durante la época de abundancia, aunque esta cifra varía entre 5 y 20 soles. En época de escasez, se paga 40 soles en promedio, cantidad que puede variar entre los 20 y los 70 soles.

El mayor precio por saco, pagado por las vendedoras de «fruto verde», se debe a que ellas ofertan aguajes de diferentes tipos (amarillo, color y shambo), cuyas materias primas tienen diferentes precios. Por su parte, las vendedoras de masa generalmente necesitan un solo tipo de saco de aguaje, el amarillo, que tiene menor precio.

Los rangos de los precios de las épocas de abundancia y de escasez son explicables a partir del hecho de que, en ambas épocas, puede haber, aunque relativa, escasez del producto. Esto se debe, principalmente, a la rutina del tráfico de las lanchas y también a una especie de regulación por los propios extractores, que no extraen frutos cuando observan que el precio se encuentra muy bajo, lo que provoca un desabastecimiento artificial.

En conjunto, las vendedoras de masa venden un total de 63 sacos diarios, lo que, en promedio, arroja una cantidad de tres sacos diarios vendidos por cada una de ellas. El $48 \%$ vende dos sacos diarios y el $24 \%$, cuatro. A su vez, las vendedoras de «fruto verde» venden un total de 61 sacos diarios, que, en promedio, significa dos sacos vendidos por cada una. El 50\% vende un saco diario y el $27 \%$, dos.

\subsection{Economía de la comercialización del aguaje}

Con los datos de las Tablas 5, 6, 7 y 8 se puede calcular el ingreso mensual promedio de las vendedoras de masa y de «fruto verde» de aguaje, tanto en época de abundancia como en época de escasez.

Para las vendedoras de masa, se tiene:

\section{INGRESOS}

3 sacos vendidos/día x 22 bolsas de masa/saco $=66$ bolsas de masa vendidas/día. 66 bolsas x $1 \mathrm{sol} /$ bolsa $=66$ soles en época de abundancia.

66 bolsas x 3 soles/bolsa $=198$ soles en época de escasez.

\section{EGRESOS}

3 sacos comprados/día x 9 soles $=27$ soles en época de abundancia. 
3 sacos comprados/día x 37 soles $=111$ en época de escasez.

Si consideramos 5 soles por gastos de transporte, derechos de mercado y otros gastos, tenemos:

\section{UTILIDAD}

$66-(27+5)=34$ soles/día $\times 30=1020$ soles/mes en época de abundancia.

$198-(111+5)=82$ soles/día x $30=2460$ soles/mes en época de escasez.

Para las vendedoras de «fruto verde», se tiene:

\section{INGRESOS}

2 sacos vendidos/día $\times 16$ bandejitas/saco $=32$ bandejitas vendidas/día.

$32 \times 1$ sol/bandejita $=32$ soles en época de abundancia.

$32 \times 5$ soles/bandejita $=160$ soles en época de escasez.

\section{EGRESOS}

2 sacos comprados/día $\times 10$ soles $=20$ soles en época de abundancia.

2 sacos comprados/día $\times 40$ soles $=80$ soles en época de escasez.

Si consideramos 5 soles por gastos de transporte, derechos de mercado y otros gastos, tenemos:

\section{UTILIDAD}

$32-(20+5)=7$ soles $/$ día $\times 30=210$ soles $/$ mes en época de abundancia. $160-(80+5)=75$ soles/día x $30=2250$ soles $/$ mes en época de escasez.

Como se observa, los ingresos obtenidos por venta de masa en época de abundancia son dos veces y media mayores que el ingreso mínimo de un trabajador de la actividad privada (410 soles). Pero los resultados observados para «fruto verde» nos indican que la utilidad es menor, lo que, al parecer, se debe a que falta precisar la información obtenida.

En cuanto a las utilidades obtenidas durante la época de escasez, en ambas actividades, se observa que se supera largamente el salario de un profesor universitario de una universidad nacional, que tenga la categoría de principal y se dedique a la docencia de manera exclusiva.

Asimismo, con los datos de las Tablas 5 y 6 , se puede deducir el consumo mensual de frutos de aguaje para estos dos rubros de la siguiente manera: 63 sacos de consumo diario para masa, sumados a 61 sacos diarios para «fruto verde» arrojan un total de 
124 sacos diarios. Esta cantidad, multiplicada por los 30 días de un mes, arroja un total de $3720 \mathrm{sacos} / \mathrm{mes}$ que, multiplicados por un peso promedio de $40 \mathrm{~kg} / \mathrm{saco}$, nos permiten calcular un total de $148,8 \mathrm{t}$.

Con estos datos, también se puede deducir el número de palmeras de aguaje cortado mensualmente para abastecer estas dos actividades, pues, como se conoce, cada palmera produce, en promedio, $138 \mathrm{~kg}$ de frutos (Rojas, 1985). Entonces, solo basta dividir el total (148 $800 \mathrm{~kg}$ ) entre la producción por planta, lo cual nos permite estimar un valor de 1078 palmeras de aguaje cortadas por mes.

Con respecto a la cantidad de sacos procesados para la elaboración de masa, de las 21 vendedoras de este producto, $29 \%$ elabora masa con todos los sacos comprados, mientras que el $71 \%$ restante, además de masa, también elabora otros productos, tal como fruta madura, aguajina (masa diluida en agua y azúcar) y curichi (aguajina congelada colocada en bolsa plástica). Esto demuestra que el mercado de masa no es muy amplio y, en vista de que la masa se oxida fácilmente y no se puede almacenar por más de tres días (Rojas et al., 2001), las vendedoras ofertan la masa sobrante convertida ya sea en fruta madura, ya sea en aguajina, o ya sea en curichi. Ello se debe a que dichos productos tienen mayor demanda. Como se ve, de esta manera, las vendedoras no pierden su inversión, pues estos productos ofrecen un mayor margen de ganancia, aunque exigen mayor tiempo para ser vendidos.

En el caso de las vendedoras de «fruto verde», se observa que ellas venden todos los sacos como tales, puesto que no necesitan transformarlos en otro subproducto. Este hecho demostraría una mayor preferencia del público por comprar aguaje en ese estado.

\subsection{Mercado, rendimiento, procedencia de aguajes de mejor calidad y ecotipo preferido}

El mercado de masa de aguaje se distribuye de la siguiente manera: el $81 \%$ vende al público en general y el $19 \%$ vende tanto al público en general como a las chupeterías de la ciudad. Pero, de las 21 vendedoras de masa, 18 de ellas se ocupan exclusivamente del consumo de Iquitos, mientras que 3 de ellas, además de vender en Iquitos, exportan su producto a otras ciudades del Perú, como Pucallpa, Tarapoto y Lima (Tabla 7).

Estos datos confirman las afirmaciones realizadas por Padoch (1988) y permiten observar, por otro lado, que, desde su publicación del año citado, el mercado de masa no se ha expandido. 
El mercado para «fruto verde» es exclusivo del público de la ciudad de Iquitos (Tabla 8). Sin embargo, actualmente, es común observar la venta de «fruto verde» en la mayoría de los mercados y supermercados de Lima. En esta ciudad, el producto se oferta a un precio de dos soles $/ \mathrm{kg}$. No es muy claro si los productos proceden de Iquitos, Pucallpa o Tarapoto.

La venta de masa se realiza en bolsas plásticas comerciales cuya capacidad es de un cuarto de kilo. Pero, debido a que las bolsas son llenadas con masa hasta el límite de su capacidad, estas pesan, en promedio, 600 gr en época de escasez y 700 gr en época de abundancia. Estas bolsas son denominadas «bolsa con cabeza» por las «maseras».

De un saco de aguaje, se obtienen, en promedio, 22 bolsas de masa. Esta cantidad varía entre 12 y 40 . El 57\% de las vendedoras señala que un saco permite producir entre 12 y 20 bolsas; $19 \%$, que permite producir entre 21 y 30 bolsas y el $19 \%$ no sabe cuántas bolsas son obtenidas de un saco de aguaje (Tabla 7). El desconocimiento del número de bolsas que se obtiene de un saco es explicable por los niveles de instrucción de las vendedoras y por el simple razonamiento de que, si se obtiene ganancias, entonces no es relevante estimar el rendimiento de la materia prima.

La venta de «fruto verde» se realiza en bandejitas plásticas de diferentes tamaños. De un saco de aguaje se obtiene, en promedio, 16 bandejitas, aunque dicha cantidad pueda variar desde 10 hasta 40 bandejitas. El 63\% de las vendedoras opina que, de un saco, se obtienen entre 10 y 20 bandejitas, y el 31\% no sabe cuántas bandejitas se obtienen de un saco. Tanto estas vendedoras como las «maseras» no se preocupan por conocer o analizar el negocio, siempre y cuando obtengan ganancias.

El $81 \%$ de las vendedoras de masa y el $67 \%$ de las vendedoras de «fruto verde» no saben cuántos frutos contiene un saco de aguaje. Para el 19\% de las vendedoras de masa, un saco contiene, aproximadamente, 825 frutos, mientras que para el $33 \%$ de las vendedoras de «fruto verde» un saco contiene, aproximadamente, 720 frutos (Tablas 7 y 8). Estos datos confirman nuestra apreciación de que no existe un interés por conocer en detalle los pormenores del negocio.

Los sacos de aguaje no están constituidos por frutos de un solo racimo. Por tal razón, existen frutos en diferentes estadios de maduración, que se evidencian cuando se ablanda el mesocarpo para comer los frutos o para preparar masa con ellos. Este hecho constituye una pérdida para las vendedoras de masa. Así, ocurre que para el $52 \%$ de las vendedoras $1 / 4$ de saco de frutos no ha madurado cuando se prepara la masa. Para el $19 \%$ eso ocurre con $1 \frac{1}{2}$ saco (Tabla 7). 
Tabla 7: Mercado, rendimiento del producto, precios de venta, procedencia de aguajes de mejor calidad y ecotipo preferido para masa.

\begin{tabular}{|c|c|c|c|c|c|c|c|c|c|c|c|}
\hline Mercado & AQV & $\begin{array}{c}\mathrm{N}^{\circ} \\
\mathrm{BM} / \mathrm{S}\end{array}$ & $\begin{array}{l}\mathrm{N}^{\circ} \\
\mathrm{F} / \mathrm{S}\end{array}$ & FNM & $\begin{array}{l}\mathrm{P} / \mathrm{BM} \\
\mathrm{A}^{\prime} \mathrm{E}\end{array}$ & & QCV & $\begin{array}{c}\text { POC } \\
\text { (en soles } \\
\text { or balde } \\
\text { o kg.) }\end{array}$ & $\begin{array}{l}\text { A } \\
\text { M } \\
\text { C } \\
\text { días) }\end{array}$ & PMC & EP \\
\hline \multirow[t]{14}{*}{ Belén } & pg & 20 & ns & $1 / 4$ saco & 1 & 2 & $\mathrm{Iq}$ & & $\mathrm{nt}$ & M & Am \\
\hline & $\mathrm{pg}$ & 18 & ns & $1 / 2$ saco & 1 & 2 & $\mathrm{Iq}$ & & 3 & A & Am \\
\hline & pg & 20 & ns & $1 / 4$ saco & 1 & 2 & $\mathrm{Iq}$ & & 3 & ns & Am \\
\hline & $\mathrm{pg}$ & 20 & ns & $1 / 4$ saco & 1 & 3 & Iq-p-t-1 & $30 / b$ & 1 & $\mathrm{M}$ & Am \\
\hline & pg & 20 & ns & $1 / 4$ Saco & 1 & 1 & $\mathrm{Iq}$ & & 1 & M-T & Am \\
\hline & pg-ch & 25 & 700 & $20-30$ & 1 & 2 & Iq-1 & $5 \mathrm{~kg}$ & 3 & I-M & Am \\
\hline & pg-ch & 30 & ns & ns & 1 & 3 & $\mathrm{Iq}$ & & 1 & $\mathrm{M}$ & Am \\
\hline & pg & 30 & ns & 1 balde & 1 & 1 & $\mathrm{Iq}$ & & $\mathrm{nt}$ & M & Am \\
\hline & pg & 20 & ns & $1 / 2$ saco & 1 & 1 & $\mathrm{Iq}$ & & $\mathrm{nt}$ & ns & Am \\
\hline & pg & 15 & 1000 & $1 / 4$ Saco & 1 & 3 & $\mathrm{Iq}$ & & 2 & $\mathrm{M}$ & Am \\
\hline & pg-ch & 40 & ns & ns & 1 & 3 & $\mathrm{Iq}$ & & 1 & I-M & Am \\
\hline & $\mathrm{pg}$ & ns & 1000 & $1 / 4 \mathrm{Saco}$ & 1 & 3 & Iq & & 6 & M & Am \\
\hline & pg & 20 & ns & $1 / 4$ saco & 1 & 2 & $\mathrm{Iq}$ & & 1 & $\mathrm{M}$ & Am \\
\hline & pg & 25 & 600 & $1 / 4$ saco & 1 & 2 & Iq-1 & $5 \mathrm{~kg}$ & 1 & ns & Am \\
\hline \multirow[t]{3}{*}{ Modelo } & pg-ch & 18 & ns & $1 / 4$ saco & 1 & 2 & $\mathrm{Iq}$ & & 1 & M & Am \\
\hline & pg & ns & ns & ns & 1 & 2 & $\mathrm{Iq}$ & & - & ns & Am \\
\hline & pg & ns & ns & $1 / 2$ saco & 1 & 2 & $\mathrm{Iq}$ & & 5 & M & Am \\
\hline \multirow[t]{2}{*}{ La Norteñita } & pg & 20 & ns & $1 / 2$ saco & 1 & 2 & $\mathrm{Iq}$ & & 2 & M & Am \\
\hline & pg & 15 & $\mathrm{~ns}$ & $1 / 4 \mathrm{Saco}$ & 1 & 2 & $\mathrm{Iq}$ & & 1 & ns & Am \\
\hline Clavero & $\mathrm{pg}$ & 12 & ns & ns & 1 & 2 & $\mathrm{Iq}$ & & 1 & M & Am \\
\hline Morona Cocha & pg & ns & ns & $1 / 4 \mathrm{Saco}$ & 1 & 1 & $\mathrm{Iq}$ & & $\mathrm{nt}$ & M & Am \\
\hline
\end{tabular}

$\mathrm{AQV}=$ A quiénes se vende.

$\mathrm{BM} / \mathrm{S}=$ Bolsas de masa/saco.

$\mathrm{F} / \mathrm{S}=$ Frutos/saco.

$\mathrm{FNM}=$ Cantidad de frutos que no maduran.

$\mathrm{P} / \mathrm{BM}=$ Precio/bolsa de masa .

$\mathrm{A}^{\prime}=$ Abundancia.

$\mathrm{E}=$ Escasez.

$\mathrm{QCV}=$ A qué ciudades se vende.

$\mathrm{POC}=$ Precio de venta para otras ciudades.

$\mathrm{AMC}=$ Almacenamiento de masa en congelación.

$\mathrm{PMC}=$ Procedencia de mejor calidad

$\mathrm{EP}=$ Ecotipo preferido.

$\mathrm{pg}=$ Público en general. ch $=$ Chupeterías.

ns $=$ No sabe.

$\mathrm{Iq}=$ Iquitos.

$\mathrm{p}=$ Pucallpa.

$\mathrm{t}=$ Tarapoto.

$1=$ Lima.

$\mathrm{nt}=$ No tiene.

$\mathrm{M}=$ Río Marañón.

$\mathrm{A}=$ Río Amazonas.

$\mathrm{T}=$ Río Tigre.

$\mathrm{I}=$ Río Itaya.

Am $=$ Amarillo. 
Una bolsa de masa cuesta un sol en época de abundancia y, en época de escasez, su costo varía desde un sol hasta tres soles. Cuando, en época de escasez, se vende al mismo precio que en época de abundancia, la bolsa contiene menos cantidad; cuando se vende a mayor precio, esto se debe a que se mantiene la cantidad de masa.

Las vendedoras de masa que exportan a otras ciudades del Perú venden su producto a cinco soles el kilo. Una de ellas oferta su mercadería en baldes de plástico, cuya medida corresponde a un balde de plástico que originalmente contenía $10 \mathrm{~kg}$ de manteca de palma aceitera (Elaeis guianensis). Calculamos que en este balde se ofrece 8 $\mathrm{kg}$ de masa. El precio de este es de 30 soles (Tabla 7).

Las vendedoras de «fruto verde» comercializan el producto en bandejitas plásticas de tamaño pequeño, que contienen aproximadamente 36 frutos, cifra que puede variar entre los 16 y 65 frutos. Esta variación está en función del tamaño del fruto, del ecotipo y de la época (de abundancia o de escasez). Sin embargo, el $50 \%$ de las vendedoras coloca entre 26 a 35 frutos por bandejita.

El precio promedio por bandejita en época de abundancia es de un sol, aunque ello pueda variar entre medio y dos soles. El 17\% de las vendedoras oferta a medio sol; el $60 \%$, a un sol y el $23 \%$, a dos soles.

El precio promedio por bandejita en época de escasez es de cinco soles. Esta cifra varía entre dos y siete soles. El 17\% de las vendedoras oferta a dos soles; el 27\%, a tres soles; el $10 \%$, a cuatro soles; el $43 \%$, a cinco soles y el $3 \%$, a siete soles (Tabla 8). La variación del precio por bandejita -entre dos y tres soles- en época de escasez se debe a la disminución del número de frutos. Si el precio es alto, ello es porque el número de frutos es constante.

Un problema serio, que encuentran tanto las vendedoras de masa como de «fruto verde», está relacionado con el almacenaje del producto. Las primeras conservan la masa en refrigeradoras o congeladoras, lo cual ocurre desde uno a seis días. Para el $43 \%$ de ellas, la masa solo puede conservarse un día, mientras que el 14\% opina que se conserva hasta tres días. Similar opinión tienen los propietarios de chupeterías (Rojas et al., 2001).

Los aguajes vendidos como «fruto verde» se conservan si se les esparce en el piso. Allí pueden estar de uno a seis días. El 47\% de las vendedoras opina que el «fruto verde» se conserva por dos días y el $33 \%$, por tres. De acuerdo con una vendedora, la conservación puede ocurrir hasta por un lapso de seis días cuando los frutos son recién cosechados. Cuando los frutos son conservados en los mismos sacos, estos se 
Tabla 8: Mercado, rendimiento del producto, precio de venta y procedencia de aguajes de mejor calidad de «fruto verde».

\begin{tabular}{|c|c|c|c|c|c|c|c|c|}
\hline Mercado & AQV & $\begin{array}{c}\text { Bandejitas } \\
\mathbf{x} \\
\text { saco }\end{array}$ & $\begin{array}{c}\mathrm{N}^{\circ} \\
\text { frutos } \mathrm{x} \\
\text { bandejita }\end{array}$ & $\begin{array}{c}\mathrm{N}^{\circ} \\
\text { frutos } x \\
\text { saco }\end{array}$ & $\begin{array}{c}\text { Precios/l } \\
\mathbf{A}^{\prime}\end{array}$ & $\begin{array}{l}\text { dejitas } \\
\text { E }\end{array}$ & $\begin{array}{c}\text { D } \\
\text { (días) }\end{array}$ & $\begin{array}{c}\text { Procedencia } \\
\text { de mejor } \\
\text { calidad }\end{array}$ \\
\hline \multirow[t]{23}{*}{ Belén } & $\mathrm{Pg}$ & 15 & 35 & 200 & 0,5 & 5 & 3 & M \\
\hline & $\mathrm{Pg}$ & 15 & 30 & 700 & 1 & 5 & 3 & M \\
\hline & $\mathrm{Pg}$ & 16 & 40 & ns & 1 & 5 & 3 & M-A \\
\hline & $\mathrm{Pg}$ & ns & 30 & 450 & 1 & 5 & 2 & M-A-I \\
\hline & $\mathrm{Pg}$ & 12 & 30 & 700 & 1 & 5 & 2 & A-I \\
\hline & $\mathrm{Pg}$ & ns & 34 & $\mathrm{~ns}$ & 1 & 5 & 3 & ns \\
\hline & $\mathrm{Pg}$ & ns & 65 & ns & 1 & 3 & 2 & ns \\
\hline & $\mathrm{Pg}$ & 13 & 40 & 600 & 2 & 7 & 2 & M \\
\hline & $\mathrm{Pg}$ & 15 & 28 & 1600 & 2 & 5 & 1 & ns \\
\hline & $\mathrm{Pg}$ & 15 & 30 & ns & 2 & 4 & 2 & $\mathrm{M}$ \\
\hline & $\mathrm{Pg}$ & 15 & 33 & ns & 1 & 3 & 2 & M \\
\hline & $\mathrm{Pg}$ & 10 & 21 & ns & 1 & 5 & 3 & M-I-N \\
\hline & $\mathrm{Pg}$ & 12 & 56 & 672 & 2 & 5 & 2 & M \\
\hline & $\mathrm{Pg}$ & 20 & 20 & $\mathrm{~ns}$ & 2 & 5 & 3 & M-A-I \\
\hline & $\mathrm{Pg}$ & 12 & 20 & ns & 0,5 & 2 & 2 & M \\
\hline & $\mathrm{Pg}$ & ns & 16 & ns & 1 & 3 & 3 & M \\
\hline & $\mathrm{Pg}$ & ns & 45 & $\mathrm{~ns}$ & 1 & 3 & 3 & M \\
\hline & $\mathrm{Pg}$ & 40 & 30 & 900 & 1 & 5 & 3 & M \\
\hline & $\mathrm{Pg}$ & 10 & 50 & $\mathrm{~ns}$ & 1 & 2 & 2 & M \\
\hline & $\mathrm{Pg}$ & ns & 30 & ns & 1 & 3 & 2 & M \\
\hline & $\mathrm{Pg}$ & 18 & 50 & ns & 2 & 5 & 2 & M \\
\hline & $\mathrm{Pg}$ & ns & 30 & ns & 1 & 2 & 2 & M \\
\hline & $\mathrm{Pg}$ & 12 & 50 & 700 & 1 & 2 & 6 & M \\
\hline \multirow[t]{7}{*}{ Modelo } & $\mathrm{Pg}$ & ns & 29 & $\mathrm{~ns}$ & 1 & 4 & 1 & M \\
\hline & $\mathrm{Pg}$ & 22 & 30 & $\mathrm{~ns}$ & 0,5 & 3 & 2 & M \\
\hline & $\mathrm{Pg}$ & ns & $\mathrm{ns}$ & 680 & 2 & 5 & 1 & M \\
\hline & $\mathrm{Pg}$ & 20 & 28 & $\mathrm{~ns}$ & 1 & 2 & 1 & A \\
\hline & $\mathrm{Pg}$ & 15 & 32 & ns & 0,5 & 3 & 1 & $\mathrm{~N}$ \\
\hline & $\mathrm{Pg}$ & 20 & 48 & ns & 1 & 4 & 3 & $\mathrm{M}$ \\
\hline & $\mathrm{Pg}$ & 18 & 63 & ns & 0,5 & 3 & 2 & $\mathrm{~N}-\mathrm{A}$ \\
\hline
\end{tabular}

$\mathrm{AQV}=\mathrm{A}$ quiénes se vende, $\mathrm{Pg}=$ Público en general, $\mathrm{A}^{\prime}=$ Abundancia, $\mathrm{E}=$ Escasez, $\mathrm{D}=$ Durabilidad de frutos de aguaje, $n s=$ No sabe, $M=$ Río Marañón, $A=$ Río Amazonas, $\mathrm{I}=$ Río Itaya, $\mathrm{N}=$ Río Nanay. 
maduran rápidamente, como máximo en dos días, pues el calor que se genera en el saco acelera la maduración.

Padoch (1988) opina que los frutos del aguaje pueden permanecer frescos por solamente cinco o seis días, mientras que, para Flores (1997), los frutos pueden permanecer de esta manera por siete días después de la cosecha. Esto solo puede ocurrir cuando los frutos están esparcidos en el piso, pero, si los frutos se encuentran en sacos, el tiempo de conservación es menor.

El ecotipo preferido para preparar masa de aguaje es el amarillo (Tabla 7), pues es el color característico de la masa y de las bebidas preparadas con ella. El público no compra masa de aguaje que sea de otro color. Por lo que respecta al «fruto verde», este es ofertado en todos los ecotipos existentes (amarillo, color y shambo), aunque cabe indicar que se observa una mayor preferencia por los ecotipos color y shambo.

En cuanto a la procedencia de frutos de mejor calidad, el 57\% de las vendedoras de masa y el $63 \%$ de las de «fruto verde» opinan que los mejores frutos proceden del río Marañón. El 24\% de las vendedoras de masa y el 10\% de las de «fruto verde» respondieron que no saben cuál es la procedencia de los frutos de mayor calidad.

La calidad en el aguaje está determinada por la maduración de los frutos, porque estos no tengan «pique»o «piojillo», porque no se vuelvan de color negro y porque sean grandes y con un mesocarpo grueso («carnudos»).

\subsection{Proceso para elaborar masa de aguaje}

Después de comprar los frutos en sacos a los diferentes mayoristas de la ciudad o directamente a las lanchas que acoderan en Iquitos, lo primero que hacen las «maseras» es colocarlos en bandejas de plástico. Luego «deschipan» o «despipan» todos los frutos. Así se le llama a la actividad que consiste en desprender manualmente el pedúnculo, el cáliz y la corola secos, que permanecen unidos al fruto después de la cosecha. Después lavan los frutos con escobillas para quitar la tierra y la suciedad que normalmente tienen los frutos. Posteriormente, agregan agua fría hasta cubrir los frutos y los dejan hasta el atardecer.

Aproximadamente a las $6 \mathrm{pm}$., se cambia el agua fría por agua caliente $\left(40\right.$ a $\left.70^{\circ} \mathrm{C}\right)$ con la finalidad de ablandar los frutos, que son dejados hasta el amanecer del día siguiente. A partir de las 3 am., en algunos casos, y de 4 a 5 am., generalmente, se retira el agua y se procede a machacar los frutos con un mazo de madera. Esto ocurre en el caso de que se observe que los frutos no están muy suaves. En caso contrario, se 
amasa con la mano. En este último caso, la operación se llama «chapear». Esta labor se realiza por espacio de 30 minutos como mínimo.

La siguiente etapa, la más difícil, consiste en eliminar las semillas y la cáscara, pues esta última corta las manos como si fuese una hoja de afeitar. La eliminación de la cáscara no es total, pues queda bastante de ella en la masa. Esta operación se denomina «preparar la masa» y demora, aproximadamente, una hora y media. Después se llenan las bolsitas de plástico de manera que estén listas para llevarlas al mercado y venderlas.

Algunas vendedoras opinan que es preferible amasar a mano. Sostienen que, cuando se machacan los frutos, se extraen los componentes amargos de la semilla que disminuyen la calidad de la masa.

\subsection{Técnica para determinar madurez fisiológica y ecotipos de aguaje}

El tiempo, la continuidad en el negocio y la variabilidad natural de los frutos de aguaje han determinado que las vendedoras de «masa» y «aguaje verde» desarrollen técnicas para determinar la madurez y los ecotipos de aguaje que compran.

Para determinar si un fruto de aguaje está suficientemente maduro, las vendedoras cortan la fruta en cualquier parte mientras la compran y dejan expuesta la semilla para observar el color de la misma. Si la semilla presenta un color blanquecino, la fruta no está fisiológicamente madura (estos frutos se denominan «shambo azul»). Ante esta situación, las vendedoras proceden a regatear el precio. Finalmente, siempre pagan un precio menor que el propuesto inicialmente. Si la semilla presenta color entre marrón y negro, eso significa que el fruto está fisiológicamente maduro.

Para determinar el ecotipo de aguaje, las compradoras raspan la cáscara del fruto de manera que dejan expuesto el mesocarpo (denominado «carne del fruto»). Si el mesocarpo es de color amarillo, esperan entre 5 y 7 minutos para determinar de qué tipo de color amarillo se trata. Si el color se mantiene firme, el aguaje es denominado «aguaje amarillo amarillo»; si se pierde un poco el color, lo denominan «aguaje amarillo posheco o pálido» y si el aguaje adquiere un color entre oscuro y negruzco, lo denominan «aguaje amarillo oscuro». El precio de los frutos varía en función de esta jerarquía. El más costoso es el «amarillo amarillo», el cual es preferido para la elaboración de chupetes y masa.

Si el mesocarpo es de color rojo, las vendedoras lo cortan hasta la semilla; si tiene el color rojo solo en la parte superficial, el aguaje se denomina «aguaje color» y si todo el mesocarpo es de color rojo, entonces el fruto se denomina «aguaje shambo». 
El «aguaje shambo» es el más apreciado y siempre cuesta más. En el año 2000, se llegó a pagar hasta 100 soles por un saco.

\subsection{Percepción sobre el futuro de la especie}

De las 21 personas que se dedican a vender masa, $81 \%$ opinó que no se debería cortar la palmera, pues así se depreda el bosque; el 19\% no opinó al respecto. A su vez, el $43 \%$ de las vendedoras cree que los frutos del aguaje no van a faltar en el mercado de Iquitos. Ello se debe a que este grupo conoce que ahora se suele subir el costo de la palmera para cosechar sus frutos. De otro lado, el 33\% cree que sí va a faltar el aguaje porque se cortan muchos troncos; mientras que el $14 \%$ opina que es posible que este falte.

El $40 \%$ de las vendedoras de «fruto verde» cree que nunca van a faltar los frutos, ya sea porque conocen que ahora se está sembrando una cantidad suficiente de ellos, ya sea porque estos existen en abundancia o porque su producción se encuentra sectorizada en la selva. Otro $40 \%$ cree que, en algún momento, los frutos van a faltar por la única razón de que son cortados, mientras que un $20 \%$ cree que posiblemente estos falten.

En síntesis, se puede afirmar que el $47 \%$ de las «maseras» y el $60 \%$ de las vendedoras de «fruto verde» percibe, en la forma de extracción, problemas en cuanto al abastecimiento futuro de frutos de aguaje. Sin embargo, el $43 \%$ de las «maseras» y el $40 \%$ de vendedoras de «fruto verde» no perciben un problema debido al sembrado de plantones y la subida del costo del tronco.

Ambas percepciones son muy alentadoras, pues las dos convergen en un mismo punto: la corta de la palmera, lo cual es muy ventajoso por la sensibilidad del tema. Cualquier proyecto que enfoque este tema tendrá asegurado el éxito, pues contará con el apoyo total de estos dos sectores.

La corriente de opinión existente ahora difiere de la opinión del año 1988, cuando se pensaba que los aguajales de la región eran inagotables y, por lo tanto, se consideraba innecesaria su plantación (Padoch, 1988).

\section{BIBLIOGRAFÍA}

BALICK, M.J. 1979. Amazonian oil palms of promise: a survey. En: The New York Botanical Garden. Economic Botany 33(1) 11-28. 
FLORES, S.P. 1997. Cultivos de frutales nativos amazónicos. Manual para el Extensionista. Lima: Tratado de Cooperación Amazónica. 370 pp.

FLORES, P. S.; GÓMEZ, R. E.; KALLIOLA, R. 1998. Características generales de la zona de Iquitos. En: Kalliola, R. y Flores, P. S. (ed.). Geoecología y desarrollo amazónico, estudio integrado en la zona de Iquitos, Perú. Turku (Finlandia): Turunyliopisto. $544 \mathrm{pp}$.

HENDERSON, A. 1995. The palms of the Amazon. New York: Oxford University Press, 361pp.

HENDERSON, A. ; GALEANO, G. ; BERNAL, R. 1995. Field guide to the palms of the Americas. New Jersey (USA): Princeton University Press. 352 pp.

INEI. 1993. Resultados definitivos de los IX censos nacionales de población, IV de Vivienda y I de comunidades nativas. Lima: INEI.

MARENGO, J. A. 1998. Climatología de la zona de Iquitos, Perú. En: Kalliola, R. y Flores, P. S. (ed.). Geoecología y desarrollo amazónico, estudio integrado en la zona de Iquitos, Perú. Turku (Finlandia): Turunyliopisto. 544 pp.

OJEDA DE HAYUM, P. 1994. Diagnóstico etnobotánico y comercialización del Morete Mauritia flexuosa (Arecaceae) en la zona del alto Napo, Ecuador. En: Alarcón, R.; Mena, P. A. y Soldi (ed.). Etnobotánica, valoración económica y comercialización de recursos florísticos silvestres en el Alto Napo, Ecuador. Quito: Moya editores. 204 pp.

PADOCH, C. 1988. Aguaje (Mauritia flexuosa L.f) in the economy of Iquitos, Perú. En: Balick, M. (ed.). The palm-tree of life: Biology, utilization and conservation. Advances in Economic Botany V6. New York: Botanical Garden. 282 pp.

ROJAS, R. R. 1985. Ensayos de germinación con semillas de 5 especies de palmeras aplicando 10 tratamientos pre-germinativos y ensayos de cosecha con 7 métodos. Iquitos (Perú): Universidad Nacional de la Amazonía Peruana. Tesis para Ingeniero Forestal. $110 \mathrm{pp}$.

ROJAS, R. R.; SALAZAR, J. C. F.; LLERENA, F. C. H.; RENGIFO, S. C.; OJANAMA, V. J.; MUÑOZ, I. V.V.; LUQUE, S. H.; SOLIGNAC, R. D.; TORRES, N. D.; PANDURO, R.F de M. 2001. Industrialización primaria del aguaje (Mauritia flexuosa L.f) en Iquitos-Perú. En prensa. 
RUIZ, M. J. 1991. El aguaje, alimento del bosque amazónico. En: Temas forestales $\mathrm{N}^{\circ}$ 8. Pucallpa (Perú): Cotesu. 28 pp.

1993. Alimentos del bosque amazónico: una alternativa para la protección de los bosques tropicales. Montevideo (Uruguay): UNESCO/ORCYT. $225 \mathrm{pp}$.

STORTI, E. F. 1993. Biología floral de Mauritia flexuosa Lin. Fil. na regiao de Manaus, Am, Brasil. En: Acta amazónica 23(4):371-381. 